\title{
Efecto de la edad relativa en la élite del balonmano femenino español y su relación con aspectos antropométricos, de rendimiento y velocidad de lanzamiento
}

\section{Relative age effect in Spanish elite of female handball and its relationship with anthropometry, physical performance and throwing speed}

\section{Efeito relativo da idade na élite do andebol feminino espanhol e sua relação com aspectos antropométricos, desempenho e velocidade de arremesso}

\author{
Ferragut, C. ${ }^{1}$, Vila, H. ${ }^{2,3}$, Fernández-Romero, J.J. ${ }^{3}$, Saavedra-García, M.A. ${ }^{3}$ \\ ${ }^{1}$ Departamento de Ciencias Biomédicas. Universidad de Alcalá, ${ }^{2}$ Facultad de Educación y Ciencias del \\ Deporte. Universidade de Vigo; ${ }^{3}$ Grupo de Investigación en Ciencias del Deporte (INCIDE), \\ Departamento de Educación Física y Deportiva. Universidade da Coruña
}

\begin{abstract}
RESUMEN
El efecto de la edad relativa (RAE) es un aspecto de relevancia en el deporte en general y en balonmano en particular. Ha sido objeto de gran cantidad de estudios centrados mayoritariamente en deporte masculino. Los estudios sobre RAE en balonmano femenino, no son tan numerosos, y no aclaran el efecto de la misma en esta modalidad deportiva. Por ello, el objetivo de este estudio fue determinar la existencia de RAE en jugadoras de élite de balonmano, así como valorar dicho efecto en función de variables antropométricas, de condición física y técnico tácticas (velocidad de lanzamiento). Han sido evaluadas 136 jugadoras de balonmano de los ocho primeros equipos de división de honor. Se ha encontrado un RAE (con la prueba de chi-cuadrado) en las jugadoras de balonmano, sustentado por aquellas que formaron parte de los equipos nacionales. No se apreció RAE en las variables antropométricas, ni en las velocidades de lanzamiento con y sin interacción. La lateralidad y el Squat Jump, presentaron RAE. El RAE es importante para llegar a la selección, pero para jugar en la máxima categoría el efecto de la edad relativa no es tan importante. Son necesarios más estudios sobre el RAE en el balonmano femenino.
\end{abstract}

Palabras clave: Efecto de la edad relativa, rendimiento, antropometría, velocidad de lanzamiento, selección nacional.

\section{ABSTRACT}




\section{Ferragut et al.}

Relative age effect (RAE) is an aspect of relevance in sport in general and in handball in particular. It has been the subject of a great number of studies focused largely on men's sport. RAE studies in female handball are not so numerous, and do not clarify the effect of female handball on this sport. Therefore, the aim of this study was to establish the effect of RAE on élite handball players, as well as to assess this effect based on anthropometric variables, physical fitness and technical and tactical skills (throwing speed). 136 handball players from the first eight honour division teams have been assessed. RAE has been found (with chi-square test) in the handball players supported by those included in national teams. No RAE were found on anthropometric variables, nor on throwing speed with and without interaction. Laterality and Squat Juam presented RAE. RAE is important to reach the national team, but to play in the highest category the effect of relative age seems to lose weight Further studies on the effect of RAE on women's handball are needed.

Keywords: Relative age effect, performance, anthropometric, throwing speed, national team.

\section{RESUMO}

O efeito da idade relativa (RAE) é um aspecto de relevância no desporto em geral e no andebol em particular. Tem sido objeto de elevado número de estudos centrados em grande parte no andebol masculino. Os estudos sobre RAE no andebol feminino, não são tão numerosos, e não clarejam o efeito da mesma nesta modalidade esportiva. Por isso, o objetivo deste estudo foi determinar a existência do RAE em jogadoras de élite de andebol, assim como valorar este efeito em função das variáveis antropométricas, de condição física e técnico-táticas (velocidade de remate). Foram avaliadas 136 jogadoras de andebol das oito primeiras equipas da divisão de honra. Encontrou-se um RAE (foi usado o teste qui-quadrado) nas jogadoras de andebol sustentado pelas que participam nas seleções nacionais. Não se encontrou RAE nas variáveis antropométricas, nem na velocidade de remate com e sem interação. A lateralidade e o Squat Juam apresentaram RAE. O RAE é importante para chegar às seleções, mas para jogar na categoria máxima o RAE parece perder significado. São necessários mais estudos sobre o RAE no andebol feminino.

Palavras chave Efeito da idade relativa, rendimento, antropometria, velocidade de lançamento, seleção nacional

\section{INTRODUCCIÓN}

El balonmano es un deporte Olímpico que se juega en todo el mundo, si bien, Europa es la que aglutina el balonmano más competitivo y de mayor calidad (Roman-Seco Seco, 2015). Sus ligas, (DKB Handball Bundesliga en Alemania, Liga Sacyr ASOBAL en España, KyH liga en Hungría, Lidl Starligue en Francia), lideran la categoría masculina, en tanto que KyH liga en Hungría, Liga Națională en Rumanía, Superleague en Rusia y HTH GO Liga en Dinamarca lideran la categoría femenina. Siendo la División de Honor de España la que ocupa el décimo lugar.

El efecto de la edad relativa, relative age effect en inglés (RAE) - que establece un sesgo que favorece la selección de los deportistas nacidos en los primeros meses del año, o cerca de la fecha de corte, frente a los nacidos al final del año o lejos de la fecha de corte ha sido ampliamente estudiado y demostrado en balonmano masculino (Karcher, Ahmaidi y Buchheit, 2014; Nakata y Sakamoto, 2011; Wrang et al., 2018) $\mathrm{y}$ menos en el balonmano femenino (Bjorndal,
Luteberget, Till y Holm, 2018; Camacho-Cardeñosa et al., 2018; Schorer et al., 2009; Schorer, Wattie y Baker, 2013). A nivel del deporte español, el efecto de la edad relativa ha sido estudiado en distintas modalidades, como el baloncesto (Arrieta, TorresUnda, Gil e Irazusta, 2016; Mujika et al., 2009), en fútbol (Gutiérrez-Diaz-del-Campo, Pastor-Vicedo, Gonzalez-Villora y Contreras-Jordan, 2010; Jimenez y Pain, 2008), atletismo (Saavedra-García, GutiérrezAguilar, Sa-Marques y Fernández-Romero, 2016) y en balonmano (Gómez-López, Angosto-Sánchez, RuízSánchez y Pérez-Turpín, 2017), siendo este último analizado a nivel regional. En el deporte femenino no son suficientes los estudios que analizan el efecto de la edad relativa (Brazo-Sayavera, Martinez-Valencia, Muller, Andronikos y Martindale, 2017), representando menos de un $2 \%$ de los estudios sobre el efecto de la edad relativa (Cobley, Baker, Wattie y McKenna, 2009). En España esta tendencia no es diferente. 


\section{Cualidades físicas, técnicas y madurativas en balonmano femenino español}

Si bien son muchos los estudios que valoran la existencia del efecto de la edad relativa en selecciones nacionales (Arrieta, Torres-Unda, Gil y Irazusta, 2016; Brustio et al., 2018; Katsumata, Omuro, Mitsukawa y Nakata, 2018; Papadopoulou, Papadopoulou, Rosemann, Knechtle y Nikolaidis, 2019; Saavedra-García, Gutiérrez-Aguilar, Galatti y Fernández-Romero, 2015; Saavedra-Garcia, Matabuena, Montero-Seoane y Fernandez-Romero, 2019; Saavedra-Garcia, Gutiérrez-Aguilar, Fernández-Romero, Fernández-Lastra y EirasOliveira, 2014), no son menos, los que analizan el comportamiento de la RAE en clubes (Beals, Furtado y Fontana, 2012; Doyle y Bottomley, 2018; Katsumata et al., 2018; Lupo et al., 2019; Penna et al., 2018), si bien pocos son realizados a nivel español y menos en el balonmano femenino. En deporte escolar federado (Leite, Borges, Santos y Sampaio, 2013; OrtigosaMarquez, Reigal, Serpa y Hernandez-Mendo, 2018) y no federado (Cervera-Raga, Jimenez-Saiz y LorenzoCalvo, 2013), los estudios son menos numerosos.

Cuando se estudia la lateralidad en el efecto de la edad relativa, encontramos estudios en tenis, en los que se encuentra una sobrerrepresentación de jugadores diestros nacidos en los dos primeros trimestres del año, pero no en jugadores zurdos, $y$, a pesar de todo, considera el ser zurdo como una ventaja para llegar a la élite del tenis (Loffing, Schorer y Cobley, 2010), en el waterpolo (Barrenetxea-Garcia, Torres-Unda, Esain y Gil, 2018) donde no se encontró un efecto de la edad relativa claro (mayor representación de zurdos en segundo y cuarto trimestre del año) y una proporción de zurdos similar a la observada en la población general (10\%). En balonmano Schorer et al. (2009), indican que la ventaja y el valor de ser zurdo radica en el hecho de que hay tres o cuatro zurdos por equipo lo que significa un $21-28 \%$ de los 14 jugadores (16 en la actualidad), porcentaje superior al de la población normal (10-13\%). Así, tanto los laterales como los extremos izquierdos presentan un efecto de la edad relativa, más débil que en las posiciones de diestro.

Existen múltiples determinantes del rendimiento, una combinación de factores antropométricos, físicos y psicológicos influyen en el éxito deportivo y en los logros de los deportistas (Abbott y Collins, 2004). Los atributos físicos y fisiológicos son importantes en el éxito deportivo en algunos deportes como el baloncesto (Torres-Unda et al., 2013) en el que se encontró que el análisis antropométrico indicaba que los jugadores de élite eran más altos, pesados, y con mayor porcentaje de masas muscular. En cuanto a los aspectos fisiológicos, mostraron un mayor rendimiento en los test de salto, resistencia y agilidad. En voleibol (Melchiorri et al., 2017) informan que las variables antropométricas, el efecto de la edad relativa y el nivel de maduración no muestran diferencias significativas en adolescentes, sin embargo, en la élite (jugadores participantes en JJOO y en Campeonatos del Mundo), se encuentran diferencias en la altura, remates y bloqueos (Palao, Manzanares y Valades, 2014). Cuando se analizan futbolistas adolescentes (Hirose, 2009) se encuentran diferencias significativas en la altura, la masa ósea y la maduración, mostrando los mejores valores en el primer trimestre del año y los peores en el cuarto, estas diferencias en altura también son encontradas por (Carling, le Gall, Reilly y Williams, 2009), pero no encuentran diferencias en las variables relacionadas con el rendimiento. En las categorías inferiores del balonmano (CamachoCardenosa et al., 2018) encuentran una sobrerrepresentación significativa de jugadores nacidos en el primer semestre del año en comparación con el segundo semestre en ambos géneros, pero sólo en el caso del género femenino en jugadoras seleccionadas en las selecciones autonómicas. En el caso masculino se han encontrado diferencias significativas en la altura, altura sentados, peso, envergadura, circunferencias de brazo y pierna y velocidad de lanzamiento, sin embargo, no se han encontrado diferencias significativas entre los dos semestres del año en categoría femenina.

El RAE ha sido ampliamente estudiado, aunque menos en mujeres que en hombres, ha sido encontrado en diferentes deportes como en el fútbol de élite en los Estados Unidos y Francia, tanto en categorías inferiores de U8 a U18 (Delorme, Boiché y Raspaud, 2010; Korgaokar, Caputo, Farley y Fuller, 2014), como en jugadoras de categoría absoluta en Francia (Delorme et al., 2010) y en España (Sedano, Vaeyens y Redondo, 2015). En el atletismo español, tras analizar las mejores marcas de todos los tiempos, se encuentra un efecto de la edad relativa en categoría femenina (Saavedra-García et al., 2016) tanto en categorías absolutas como en inferiores. En deportes con balón, el estudio del efecto de la edad relativa ha sido generalizado en Israel (Lidor, Arnon, Maayan, Gershon y Côté, 2014) no encontrando efecto de la 


\section{Ferragut et al.}

edad relativa en los cuatro deportes analizados: baloncesto, balonmano (al contrario que este estudio), fútbol y voleibol. De igual modo, por ejemplo, el efecto de la edad relativa no ha sido encontrado en deportistas femeninas participantes en atletas de taekwondo olímpicas (Albuquerque et al., 2012). Estas respuestas tan dispares pueden ser debidas al menor nivel de competitividad del deporte femenino en relación al masculino, encontrando mayor efecto de la edad relativa en deportes muy practicados y pudiendo llegar a desaparecer, más fácilmente, en deportes con menor número de practicantes.

Por lo expuesto anteriormente, el objetivo de este estudio ha sido determinar la existencia del efecto de la edad relativa en jugadoras de élite del balonmano español y valorar dicho efecto en función de variables antropométricas, de condición física y en las velocidades de lanzamiento.

\section{MATERIAL Y MÉTODOS}

\section{Diseño de Investigación}

Se ha utilizado un diseño de investigación empírico, utilizando una estrategia descriptiva de tipo observacional (Ato, Lopez y Benavente, 2013). El estudio tiene un diseño cuantitativo, no experimental, de carácter descriptivo y transversal.

\section{Participantes}

La muestra, obtenida en el año 2014, y formada por

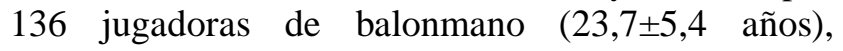
pertenecientes a los ocho primeros equipos de la División de Honor, entre las cuales se encuentran las componentes de los equipos nacionales de España (juveniles, júnior y sénior) y jugadoras de selecciones de otros países.

Todas las jugadoras y cuerpo técnico fueron informados de las pruebas que se iban a realizar, así como de los posibles riesgos que estas entrañaban. Todas ellas firmaron su consentimiento para participar en este estudio, avalado por el comité de ética de la Universidade de Vigo (Referencia 04-719). El estudio se llevó a cabo de conformidad con la Declaración de Helsinki, que establece los principios éticos fundamentales para la investigación con seres humanos (WMA, 2009; Bošnjak, 2001; Tyebkhan, 2003).

\section{Procedimientos}

Para la evaluación condicional, todas las jugadoras fueron sometidas a diversas pruebas consistentes en: 1) una evaluación antropométrica que incluyó una evaluación de la composición corporal y el somatotipo, 2) test de salto vertical tanto salto con contramovimiento (CMJ) como salto sin contramovimiento (SJ), para evaluar la fuerza explosiva del tren inferior, 3) Fuerza de prensión manual y la velocidad de lanzamiento en varias situaciones (Lanzamiento de $7 \mathrm{~m}$ sin portera, $9 \mathrm{~m}$ sin portera, 9+3 $\mathrm{m}$ sin portera, Suspensión sin portera, Lanzamiento de $7 \mathrm{~m}$ con portera, $9 \mathrm{~m}$ con portera, $9+3$ $\mathrm{m}$ con portera, Suspensión con portera).

\section{Valoración antropométrica}

Para la valoración antropométrica se siguió el protocolo de la International Society for the Advancement of Kinanthropometry (ISAK) (Ross y Marfell-Jones, 1995).

Se les tomaron las medidas durante una única sesión, de manera unilateral por el lado derecho del cuerpo. Las jugadoras fueron evaluadas con ropa ligera y sin zapatos. Las mediciones se realizaron en el siguiente orden: altura, peso, envergadura, pliegues cutáneos, perímetros y diámetros. La altura y el peso se midieron con una báscula con tallímetro (Seca, Barcelona, Spain), con una precisión de $0,01 \mathrm{~kg}$ y $0,001 \mathrm{~m}$ respectivamente. Se tomaron nueve pliegues (tricipital, subscapular, bicipital, axilar, abdominal, cresta iliaca, suprailíaco, medial del muslo y pantorrilla) con plicómetro Holtain que ejerció una presión constante de 10 g. $\mathrm{mm}^{-2}$. Los perímetros corporales (brazo relajado, brazo contraído, antebrazo, muñeca, pecho, glúteo, pantorrilla y tobillo) se midieron con una cinta inextensible Lufkin (Lufkin Executive Thinline, W606PM, USA) y los diámetros óseos (Biacromial, bicondileo, biepicondileo, biileocrestal bitrocantereo y biestiloideo) con un antropómetro GPM, Switzerland con una precisión de $0.01 \mathrm{~cm}$.

Las valoraciones fueron realizadas por un técnico nivel I de la ISAK y se tomaron dos veces (tres en el caso de los pliegues cutáneos). El error técnico de medida fue $<2 \%$ para todos los pliegues y $<1 \%$ para los perímetros y diámetros óseos.

A través de los datos antropométricos se calcularon las siguientes variables: (a) el índice de masa corporal (IMC) calculado como el peso $(\mathrm{kg})$ dividido por la 


\section{Cualidades físicas, técnicas y madurativas en balonmano femenino español}

altura al cuadrado (m), (b) La suma de cuatro y seis pliegues, (c) La masa libre de grasa (FFM) (Kg) usando el método descrito por (Lee et al., 2000), (d) El somatotipo siguiendo el método de (Carter y Heath, 1990).

\section{Salto vertical}

Cada jugadora realizó dos tipos de salto vertical en una plataforma (REF), el SJ se realizó en posición de pie con las rodillas flexionadas a $90^{\circ}$ y con las manos en la cintura para evitar el efecto de los brazos y el CMJ en el que se comenzó desde la posición de pie con los bazos en la cintura para posteriormente bajar con una flexión de rodilla rápida hasta los $90^{\circ}$ justo antes de la impulsión. Los sujetos realizaron tres intentos de cada salto con 3 minutos de descanso entre ellos y el mejor de cada uno (en términos de tiempo de vuelo) se utilizó para el posterior análisis. La altura del salto se calculó posteriormente a partir del tiempo de vuelo (Bosco, Luhtanen y Komi, 1983).

\section{Velocidad de lanzamiento}

La velocidad de lanzamiento fue determinada con una pistola radar (StalkerPro Inc., Plano), con $100 \mathrm{~Hz}$ de frecuencia de muestreo y con una sensibilidad de $0,045 \mathrm{~m} \cdot \mathrm{s}-1$, colocada detrás de la portería.

Antes de los lanzamientos, los jugadores realizaron 15 minutos de calentamiento centrado en el lanzamiento. Se realizaron dos protocolos de lanzamiento con un balón oficial de balonmano, uno con portera y otro sin portera. En cada uno se efectuaron los siguientes lanzamientos: $7 \mathrm{~m}$, desde $9 \mathrm{~m}$ parado, desde $9 \mathrm{~m}$ en apoyo con tres pasos previos y desde $9 \mathrm{~m}$ en suspensión con tres pasos previos. Se realizaron 3 lanzamientos de cada tipo, seleccionando el mejor para el posterior análisis. Se dejaron 3 minutos de descanso entre cada lanzamiento para evitar el efecto de la fatiga y para motivar a las jugadoras se les informó del resultado en cada lanzamiento.

En la valoración de la velocidad las porteras no se han incluido.

\section{Dinamometría manual}

La fuerza isométrica máxima del antebrazo fue obtenida con un dinamómetro de mano (T.K.K. 5401, Japan), con una sensibilidad de 0,1 kilogramo fuerza (Kgf). Las jugadoras fueron familiarizadas con el dinamómetro con tres repeticiones realizadas durante el calentamiento. Las jugadoras realizaron dos repeticiones máximas con la mano dominante. Para ello, se colocaron de pie con el dinamómetro paralelo al cuerpo. En esta posición se les pidió que ejercieran la mayor fuerza posible sin mover ni el brazo ni la muñeca. El mejor de los dos intentos fue empleado para el posterior análisis. Se esperó tres minutos entre intento para evitar el efecto de la fatiga.

\section{Variables de estudio}

Se han analizado las variables relativas a la pertenencia o no a las distintas selecciones de España, la fecha de nacimiento, variables antropométricas como la altura, el peso, la envergadura, el IMC, variables de juego como el puesto específico en el campo de juego, y los años de práctica del balonmano. Además, se han incluido variables relativas a la condición física, como la dinamometría de ambas manos, el salto $\mathrm{SJ}$, el salto $\mathrm{CMJ}$ y variables relativas a la condición física y al juego como el lanzamiento de $7 \mathrm{~m}$ con y sin portera, 9 metros con sin portera, $9+3 \mathrm{~m}$ con y sin portera, suspensión con y sin portera. Finalmente se miden variables antropométricas como los pliegues cutáneos, circunferencias, diámetros, longitudes, porcentajes y los pesos grasos, musculares, óseos, la endomorfía, mesomorfía y ectomorfía. Una vez obtenidos los datos se ha calculado la variable trimestre de nacimiento a partir de la fecha de nacimiento de las jugadoras, siguiendo los procedimientos en los que se considera el número de días en cada trimestre, lo que supone una corrección a la distribución de probabilidad uniforme (Delorme y Champely, 2015; Doblhammer y Vaupel, 2001; Edgar y O'Donoghue, 2005)

$$
X_{D}^{2}=\sum_{i=1}^{4} \frac{\left(n_{i}-n \cdot d_{i}\right)^{2}}{n \cdot d_{i}}
$$

con $\quad d_{1}=\frac{31+28,25+31}{365,25}, \quad d_{2}=\frac{30+31+30}{365,25}, d_{3}=$ $\frac{31+31+30}{365,25}$ y $d_{4}=\frac{31+30+31}{365,25}$. Donde $n_{i}$ es la frecuencia de nacimientos observada en el trimestre $i$. El tamaño de la muestra se representa por $n=\sum_{i=1}^{4} n_{i}$. Así los valores de $d_{1}$ a $d_{4}$ son 0,$2471 ; 0,2491 ; 0,2519$ y 0,2519 respectivamente.

La fecha de corte en el balonmano español es el 1 de enero, por tanto, se divide el año en cuatro trimestres. Así las jugadoras nacidas entre el 1 de enero y el 31 de marzo pertenecen al primer trimestre $(\mathrm{Q} 1)$, las nacidas entre el 1 de abril al 30 de junio pertenecen segundo 


\section{Ferragut et al.}

trimestre (Q2), entre el 1 de julio y el 30 de septiembre han nacido en el tercer trimestre del año (Q3) y finalmente entre el 1 de octubre y el 31 de diciembre se clasifican en el cuarto trimestre del año (Q4).

\section{Procedimiento estadístico}

Para determinar si se encuentra el efecto de la edad relativa se ha utilizado la prueba de chi-cuadrado. En caso de obtener valores significativos de chi-cuadrado se calculan los residuos estandarizados (pruebas posthoc) para determinar que trimestres se desvían significativamente de los valores esperados. Los residuos estandarizados denotan la desviación de la distribución esperada (Garson, 2010; Hancock, Young y Ste-Marie, 2011; Saavedra-García et al., 2016). Se consideran significativos del residuo estandarizado con valores mayores de 1,96 en valor absoluto. Los valores positivos indican una sobrerepresentación de nacimientos en un trimestre del año en relación al valor esperado, en tanto que los valores negativos indican una baja representación. El tamaño del efecto (TE) de la significación de chi-cuadrado se define como la raíz cuadrada del cociente entre el estadístico de chi-cuadrado $\left(\chi^{2}\right)$ y el número de casos (Albuquerque, 2013; Hancock et al., 2011). Los valores de TE de 0,1 indican un efecto pequeño, los valores en torno a 0,3 un efecto medio y valores cercanos a 0,5 representa un efecto grande (Cohen, 1988).

Para comparar las variables antropométricas, de condición física y los aspectos técnico-tácticos entre los trimestres del año se ha realizado un ANOVA, garantizando previamente la normalidad (prueba KS) y la igualdad de varianzas (Levene). En caso de no cumplirse alguno de los prerrequisitos, se aplicarán las pruebas no paramétricas de la Mediana y la prueba de Kruskal-Wallis.

Los datos han sido analizados con el SPSS 21. Se ha considerado la significación estadística cuando el pvalor es menor de 0,05 .

\section{RESULTADOS}

Distribución de las fechas de nacimiento de las jugadoras de balonmano.

La distribución por trimestre de nacimiento de las jugadoras de balonmano se presenta en la tabla 1.

\section{Tabla 1}

Efecto de la edad relativa de las jugadoras de balonmano

\begin{tabular}{|c|c|c|c|c|c|c|c|c|c|c|c|c|}
\hline \multirow{2}{*}{ Género } & \multirow{2}{*}{$\mathrm{n}$} & \multirow{2}{*}{$\chi^{2}$} & \multirow{2}{*}{ Sig. } & \multirow{2}{*}{$\mathrm{TE}$} & \multicolumn{4}{|c|}{$\mathrm{n}$} & \multicolumn{4}{|c|}{ Residuos estandarizados } \\
\hline & & & & & Q1 & $\mathrm{Q} 2$ & Q3 & Q4 & Q1 & Q2 & Q3 & Q4 \\
\hline Femenino & 136 & 8,869 & $\mathbf{0 , 0 3 4} *$ & 0,255 & 48 & 26 & 30 & 32 & $2,49 * *$ & $-1,35$ & $-0,73$ & $-0,39$ \\
\hline
\end{tabular}

*Significación estadística de la prueba de $\chi^{2}$ (Chi-cuadrado). Q1 a Q4 = Trimestres 1 a 4. TE = Tamaño del Efecto.

**Significación de los residuos estandarizados

La distribución global observada difiere significativamente de la esperada en las jugadoras de balonmano $\left(\chi^{2}=8,869 ;\right.$ g.l. $\left.=3 ; \mathrm{p}<0,035\right)$. Se aprecia una sobre representación de jugadoras nacidas en el primer trimestre del año.

\section{Distribución de las fechas de nacimiento en función de la pertenencia o no a las selecciones nacionales.}

Si se clasifica a las jugadoras en función de que hayan participado o no en las selecciones nacionales (Tabla 2), se han encontrado diferencias entre las distribuciones observadas y las esperadas en las jugadoras seleccionadas $\left(\chi^{2}=11,056 ;\right.$ g.l. $=3$; $\mathrm{p}<0,012)$, mostrándose una sobre representación de jugadoras nacidas en el primer trimestre del año, en tanto que en las jugadoras no seleccionadas no se ha encontrado un efecto de la edad relativa.

Distribución de las fechas de nacimiento en función de la lateralidad de las jugadoras.

Si se clasifica a las jugadoras en función de su lateralidad (Tabla 3), se encuentran diferencias entre las distribuciones observadas y las esperadas en las jugadoras diestras $\left(\chi^{2}=8,825 ;\right.$ g.l. $\left.=3 ; \mathrm{p}<0,033\right)$, mostrándose una sobre representación de jugadoras nacidas en el primer trimestre del año, en tanto que en las jugadoras zurdas no se han encontrado diferencias significativas. 


\section{Tabla 2}

Efecto de la edad relativa en función de si las jugadoras pertenecen o no a alguna selección nacional

\begin{tabular}{|c|c|c|c|c|c|c|c|c|c|c|c|c|}
\hline \multirow{2}{*}{ Selección } & \multirow{2}{*}{$\mathrm{n}$} & \multirow{2}{*}{$\chi^{2}$} & \multirow{2}{*}{ Sig. } & \multirow{2}{*}{$\mathrm{TE}$} & \multicolumn{4}{|c|}{$\mathrm{n}$} & \multicolumn{4}{|c|}{ Residuos estandarizados } \\
\hline & & & & & Q1 & Q2 & Q3 & Q4 & Q1 & Q2 & Q3 & Q4 \\
\hline $\mathrm{Si}$ & 73 & 11,056 & $0,011 *$ & 0,389 & 30 & 16 & 12 & 15 & $2,82 * *$ & $-0,51$ & -1.49 & $-0,79$ \\
\hline No & 63 & 2,808 & 0,422 & 0,211 & 18 & 10 & 18 & 17 & 0,62 & $-1,44$ & 0,53 & 0,28 \\
\hline
\end{tabular}

*Significación estadística de la prueba de $\chi^{2}$ (Chi-cuadrado). Q1 a Q4 = Trimestres 1 a 4 . TE = Tamaño del Efecto.

**Significación de los residuos estandarizados

\section{Tabla 3}

Efecto de la edad relativa en función de la lateralidad de las jugadoras

\begin{tabular}{|c|c|c|c|c|c|c|c|c|c|c|c|c|}
\hline \multirow{2}{*}{ Lateralidad } & \multirow{2}{*}{$\mathrm{n}$} & \multirow{2}{*}{$\chi^{2}$} & \multirow{2}{*}{ Sig. } & \multirow{2}{*}{$\mathrm{TE}$} & \multicolumn{4}{|c|}{$\mathrm{n}$} & \multicolumn{4}{|c|}{ Residuos estandarizados } \\
\hline & & & & & Q1 & Q2 & Q3 & Q4 & Q1 & Q2 & Q3 & Q4 \\
\hline Diestra & 113 & 8,992 & 0,029* & 0,282 & 41 & 20 & 25 & 27 & $2,48 * *$ & $-1,53$ & -0.65 & $-0,28$ \\
\hline Zurda & 23 & 0,538 & 0,910 & 0,153 & 7 & 6 & 5 & 5 & 0,55 & 0,11 & $-0,33$ & $-0,33$ \\
\hline
\end{tabular}

*Significación estadística de la prueba de $\chi^{2}$ (Chi-cuadrado). Q1 a Q4 = Trimestres 1 a 4. TE = Tamaño del Efecto.

**Significación de los residuos estandarizados

Distribución de las fechas de nacimiento en función del puesto específico de las jugadoras.

Si se clasifica a las jugadoras en función de su posición (Tabla 4), se encuentran diferencias entre las distribuciones observadas y las esperadas en las posiciones de pivote $\left(\chi^{2}=15,245 ;\right.$ g.l. $\left.=3 ; \mathrm{p}<0,003\right) \mathrm{y}$ portera $\left(\chi^{2}=13,457\right.$ g.l. $\left.=3 ; \mathrm{p}<0,005\right)$, mostrándose tanto en el caso de las pivotes como de las porteras una sobre representación de jugadoras nacidas en el primer trimestre del año y una baja representación en el segundo trimestre del año en el caso de las porteras. Por el contrario, en los puestos específicos de central, lateral y extremo, no se han encontrado diferencias significativas.

\section{Tabla 4}

Efecto de la edad relativa en función del puesto específico de las jugadoras

\begin{tabular}{|c|c|c|c|c|c|c|c|c|c|c|c|c|}
\hline \multirow{2}{*}{ Puesto } & \multirow{2}{*}{$\mathrm{n}$} & \multirow{2}{*}{$\chi^{2}$} & \multirow{2}{*}{ Sig. } & \multirow{2}{*}{$\mathrm{TE}$} & \multicolumn{4}{|c|}{$\mathrm{n}$} & \multicolumn{4}{|c|}{ Residuos estandarizados } \\
\hline & & & & & Q1 & Q2 & Q3 & Q4 & Q1 & Q2 & Q3 & Q4 \\
\hline Central & 20 & 2,881 & 0,410 & 0,380 & 6 & 7 & 2 & 5 & 0,48 & 0,91 & $-1,35$ & $-0,02$ \\
\hline Lateral & 28 & 1,228 & 0,746 & 0,209 & 9 & 7 & 5 & 7 & 0,79 & 0.01 & -0.77 & $-0,02$ \\
\hline Extremo & 37 & 1,685 & 0,640 & 0,213 & 6 & 9 & 11 & 11 & -1.04 & $-0,07$ & 0,55 & 0,55 \\
\hline Pivote & 19 & 15,245 & $0,002 *$ & 0,896 & 12 & 2 & 2 & 3 & $3,37 * *$ & $-1,26$ & $-1,27$ & $-0,82$ \\
\hline Portera & 32 & 13,457 & $0,004 *$ & 0,648 & 15 & 1 & 10 & 6 & $2,52 * *$ & $-2,47 * *$ & 0,68 & $-0,73$ \\
\hline
\end{tabular}

*Significación estadística de la prueba de $\chi^{2}$ (Chi-cuadrado). Q1 a Q4 = Trimestres 1 a 4. TE = Tamaño del Efecto.

**Significación de los residuos estandarizados

Correspondence to: Miguel Saavedra García. Grupo INCIDE, Departamento de Educación

Física y Deportiva, Universidade da Coruña, Avd. Ernesto Che Guevara, 121, 15179 Oleiros,

A Coruña (España). Tel: +34981167000. Email: miguel.saavedra@udc.es 


\section{Ferragut et al.}

Antropometría básica y efecto de la edad relativa.

Como se aprecia en la tabla 5, no se han encontrado diferencias significativas en las variables antropométricas básicas en función del trimestre de nacimiento. Tampoco se han encontrado diferencias en pliegues cutáneos, circunferencias, diámetros, longitudes, porcentajes y los pesos grasos, musculares, óseos, la endomorfía, mesomorfía y ectomorfía.

Tabla 5

Descripción y comparación de variables antropométricas básicas en función del trimestre del año

\begin{tabular}{|c|c|c|c|c|c|c|c|}
\hline Variable & Trimestre & $\overline{\mathrm{X}} \pm 0$ & Sig. KS & Sig. Levene & Sig. ANOVA & Sig. Mediana & Sig. KW \\
\hline \multirow[t]{4}{*}{ Altura } & Q1 & $172,45 \pm 7,51$ & 0,412 & \multirow{4}{*}{0,518} & \multirow{4}{*}{0,748} & \multirow{4}{*}{--} & \multirow{4}{*}{--} \\
\hline & $\mathrm{Q} 2$ & $172,22 \pm 6,53$ & 0,963 & & & & \\
\hline & $\mathrm{Q} 3$ & $172,25 \pm 6,52$ & 0,818 & & & & \\
\hline & $\mathrm{Q} 4$ & $170,75 \pm 7,62$ & 0,425 & & & & \\
\hline \multirow[t]{4}{*}{ Peso } & Q1 & $71,46 \pm 11,43$ & 0,830 & \multirow{4}{*}{0,048} & \multirow{4}{*}{0,176} & \multirow{4}{*}{--} & \multirow{4}{*}{--} \\
\hline & $\mathrm{Q} 2$ & $69,31 \pm 8,03$ & 0,571 & & & & \\
\hline & Q3 & $68,13 \pm 8,75$ & 0,923 & & & & \\
\hline & Q4 & $66,88 \pm 7,72$ & 0,645 & & & & \\
\hline \multirow[t]{4}{*}{ Envergadura } & Q1 & $171,79 \pm 8.03$ & 0,434 & \multirow{4}{*}{0,806} & \multirow{4}{*}{0,717} & \multirow{4}{*}{--} & \multirow{4}{*}{--} \\
\hline & Q2 & $171,73 \pm 6,94$ & 0,738 & & & & \\
\hline & $\mathrm{Q} 3$ & $173,23 \pm 8,28$ & 0,876 & & & & \\
\hline & $\mathrm{Q} 4$ & $170,77 \pm 9,04$ & 0,455 & & & & \\
\hline \multirow[t]{4}{*}{ IMC } & $\mathrm{Q} 1$ & $23,99 \pm 3,33$ & 0,067 & \multirow{4}{*}{0,208} & \multirow{4}{*}{--} & \multirow{4}{*}{0,670} & \multirow{4}{*}{0,703} \\
\hline & $\mathrm{Q} 2$ & $23,37 \pm 2,42$ & 0,466 & & & & \\
\hline & Q3 & $22,94 \pm 2,09$ & 0,510 & & & & \\
\hline & Q4 & $22,90 \pm 1,67$ & 0,481 & & & & \\
\hline
\end{tabular}

*Significación estadística

KS: Prueba de Kolmogorov-Smirnov

KW: Prueba de Kruskal-Wallis

Condición física y efecto de la edad relativa.

En la tabla 6, no se muestran diferencias significativas en la dinamometría máxima. En el salto $\mathrm{SJ}$, se encuentran diferencias significativas $(p<0,038)$, mostrando mayor capacidad de salto las jugadoras del tercer y cuarto trimestre frente a las nacidas en el primer semestre del año. En el CMJ, tampoco se aprecian diferencias significativas.

\section{Tabla 6}

Descripción y comparación de variables de condición física en función del trimestre del año.

\begin{tabular}{|c|c|c|c|c|c|c|c|}
\hline Variable & Trimestre & $\overline{\mathrm{X}} \pm 0$ & Sig. KS & Sig. Levene & Sig. ANOVA & Sig. Mediana & Sig. KW \\
\hline \multirow[t]{4}{*}{ Dinamometría máxima } & Q1 & $36,37 \pm 5,95$ & 0,988 & \multirow{4}{*}{0,035} & \multirow{4}{*}{--} & \multirow{4}{*}{0,733} & \multirow{4}{*}{0,713} \\
\hline & Q2 & $36,90 \pm 3,36$ & 0,829 & & & & \\
\hline & Q3 & $35,26 \pm 4,23$ & 0,962 & & & & \\
\hline & Q4 & $36,27 \pm 5,74$ & 0,890 & & & & \\
\hline \multirow[t]{4}{*}{ Salto SJ } & Q1 & $0,37 \pm 0,10$ & 0,299 & \multirow{4}{*}{0,254} & \multirow{4}{*}{--} & \multirow{4}{*}{$\mathbf{0 , 0 3 7 *}$} & \multirow{4}{*}{0,059} \\
\hline & Q2 & $0,36 \pm 0,11$ & 0,299 & & & & \\
\hline & Q3 & $0,39 \pm 0,10$ & 0,304 & & & & \\
\hline & Q4 & $0,43 \pm 0,09$ & 0,025 & & & & \\
\hline
\end{tabular}




\section{Cualidades físicas, técnicas y madurativas en balonmano femenino español}

\begin{tabular}{|c|c|c|c|c|c|c|c|}
\hline \multirow[t]{4}{*}{ Salto CMJ } & Q1 & $0,39 \pm 0,10$ & 0,482 & \multirow{4}{*}{0,714} & \multirow{4}{*}{--} & \multirow{4}{*}{0,129} & \multirow{4}{*}{0,060} \\
\hline & $\mathrm{Q} 2$ & $0,38 \pm 0,11$ & 0,708 & & & & \\
\hline & Q3 & $0,40 \pm 0,11$ & 0,279 & & & & \\
\hline & Q4 & $0,45 \pm 0,10$ & 0,045 & & & & \\
\hline
\end{tabular}

*Significación estadística; KS: Prueba de Kolmogorov-Smirnov; KW: Prueba de Kruskal-Wallis

Velocidad de lanzamientos en distintas situaciones de juego y efecto de la edad relativa.

Como se puede ver en la tabla 7 , no se aprecian diferencias significativas en la velocidad de lanzamiento en distintas situaciones de juego, tales como el lanzamiento de $7 \mathrm{~m}$ con o sin portero, los lanzamientos de 9 metros y 9 metros con tres pasos previos con y sin portero, así como los lanzamientos en suspensión con y sin portero.

\section{Tabla 7}

Velocidades de lanzamiento en distintas situaciones de juego (sólo jugadoras de campo).

\begin{tabular}{|c|c|c|c|c|c|c|c|}
\hline Variable & Trimestre & $\overline{\mathrm{X}} \pm 0$ & $\begin{array}{l}\text { Sig. } \\
\text { KS }\end{array}$ & $\begin{array}{c}\text { Sig. } \\
\text { Levene }\end{array}$ & $\begin{array}{c}\text { Sig. } \\
\text { ANOVA }\end{array}$ & $\begin{array}{c}\text { Sig. } \\
\text { Mediana }\end{array}$ & $\begin{array}{l}\text { Sig. } \\
\text { KW }\end{array}$ \\
\hline \multirow{4}{*}{$\begin{array}{l}\text { Lanzamiento de } 7 \mathrm{~m} \text { sin } \\
\text { portera }\end{array}$} & Q1 & $73,87 \pm 7,40$ & 0,539 & \multirow{4}{*}{0,318} & \multirow{4}{*}{0,586} & \multirow{4}{*}{--} & \multirow{4}{*}{--} \\
\hline & Q2 & $74,81 \pm 6,34$ & 0,999 & & & & \\
\hline & Q3 & $74,00 \pm 5,38$ & 0,892 & & & & \\
\hline & Q4 & $76,13 \pm 5,33$ & 0,466 & & & & \\
\hline \multirow[t]{4}{*}{$9 \mathrm{~m} \sin$ portera } & Q1 & $74,61 \pm 6,84$ & 0,039 & \multirow{4}{*}{0,883} & \multirow{4}{*}{--} & \multirow{4}{*}{0.310} & \multirow{4}{*}{0,098} \\
\hline & Q2 & $76,24 \pm 6,80$ & 0,547 & & & & \\
\hline & Q3 & $74,26 \pm 5,58$ & 0,414 & & & & \\
\hline & Q4 & $75,39 \pm 5,31$ & 0,936 & & & & \\
\hline \multirow[t]{4}{*}{$9+3 \mathrm{~m}$ sin portera } & Q1 & $79,94 \pm 6,64$ & 0,369 & \multirow{4}{*}{0,844} & \multirow{4}{*}{0,283} & \multirow{4}{*}{--} & \multirow{4}{*}{--} \\
\hline & Q2 & $82,38 \pm 7,05$ & 0,897 & & & & \\
\hline & Q3 & $79,63 \pm 6,01$ & 0,962 & & & & \\
\hline & Q4 & $82,35 \pm 5,31$ & 0,687 & & & & \\
\hline \multirow[t]{4}{*}{ Suspensión sin portera } & Q1 & $80,74 \pm 6,87$ & 0,727 & \multirow{4}{*}{0,225} & \multirow{4}{*}{0,638} & \multirow{4}{*}{--} & \multirow{4}{*}{--} \\
\hline & $\mathrm{Q} 2$ & $81,05 \pm 5,53$ & 0,758 & & & & \\
\hline & Q3 & $78,95 \pm 4,53$ & 0,690 & & & & \\
\hline & Q4 & $80,70 \pm 4,70$ & 0,856 & & & & \\
\hline \multirow{4}{*}{$\begin{array}{l}\text { Lanzamiento de } 7 \mathrm{~m} \text { con } \\
\text { portera }\end{array}$} & Q1 & $72,48 \pm 6,28$ & 0,184 & \multirow{4}{*}{0,877} & \multirow{4}{*}{0.356} & \multirow{4}{*}{--} & \multirow{4}{*}{--} \\
\hline & Q2 & $73,96 \pm 5,74$ & 0,958 & & & & \\
\hline & Q3 & $71,50 \pm 5,03$ & 0,826 & & & & \\
\hline & Q4 & $74,23 \pm 6,20$ & 0,949 & & & & \\
\hline \multirow[t]{4}{*}{$9 \mathrm{~m}$ con portera } & Q1 & $74,25 \pm 6,31$ & 0,837 & \multirow{4}{*}{0,730} & \multirow{4}{*}{0,446} & \multirow{4}{*}{--} & \\
\hline & Q2 & $75,88 \pm 5,47$ & 0,786 & & & & \\
\hline & Q3 & $74,50 \pm 4,57$ & 0,804 & & & & -- \\
\hline & Q4 & $76,35 \pm 5,48$ & 0,998 & & & & \\
\hline $9+3 \mathrm{~m}$ con portera & Q1 & $79,12 \pm 6,74$ & 0,520 & & & & \\
\hline & Q2 & $81,80 \pm 4,99$ & 0,859 & & & & \\
\hline & Q3 & $78,95 \pm 4,60$ & 0,864 & 0,335 & 0,173 & -- & -- \\
\hline & Q4 & $81,50 \pm 5,21$ & 0,996 & & & & \\
\hline Suspensión con portera & Q1 & $79,55 \pm 5,74$ & 0,426 & & & & \\
\hline & Q2 & $79,28 \pm 5,81$ & 0,864 & & & & \\
\hline & Q3 & $78,68 \pm 4,56$ & 0,832 & 0,350 & 0,955 & -- & -- \\
\hline & Q4 & $79,31 \pm 4,54$ & 0,587 & & & & \\
\hline
\end{tabular}

\footnotetext{
*Significación estadística; KS: Prueba de Kolmogorov-Smirnov; KW: Prueba de Kruskal-Wallis
} 


\section{Ferragut et al.}

\section{DISCUSIÓN}

Nuestro objetivo principal ha sido determinar la existencia del efecto de la edad relativa (utilizando la prueba de chi-cuadrado) en jugadoras de élite del balonmano español y valorar dicho efecto en función de variables antropométricas, de condición física y en las velocidades de lanzamiento. Se ha encontrado un efecto de la edad relativa en el balonmano femenino español, sostenido básicamente por las jugadoras que han jugado en las distintas categorías de la selección nacional, no encontrando un RAE significativo en las jugadoras no seleccionadas. En relación a la lateralidad, sólo hemos encontrado efecto de la edad relativa en jugadoras diestras, pero no en jugadoras zurdas. En el análisis por puesto específico, sólo las porteras y las pivotes muestran un efecto significativo, más fuerte en las pivotes que en las porteras. Además, las variables antropométricas no muestran diferencias significativas cuando se comparan en función del trimestre de nacimiento de las jugadoras, pero si se encuentran diferencias en variables relacionadas con la condición física, concretamente en el SJ, donde apreciamos un efecto de la edad relativa inverso. Finalmente, en las velocidades de lanzamiento en distintas situaciones de juego no hemos encontrado diferencias significativas.

En relación al balonmano, se ha encontrado una baja representación de jugadoras noruegas de balonmano en el cuarto trimestre del año, en los dos años en los que transcurre cada categoría (Bjorndal et al., 2018), también en el balonmano en categorías inferiores españolas se encuentra una sobrerrepresentación de jugadoras nacidas en el primer semestre del año (Camacho-Cardenosa et al., 2018). Del mismo modo, en el balonmano alemán, se ha encontrado una sobrerrepresentación de jugadoras nacidas en los dos primeros trimestres del año (Schorer et al., 2009), todos estos estudios son acordes a los datos encontrados en el presente estudio, si bien, globalmente, en el presente estudio, encontramos una sobrerrepresentación en el primer y tercer trimestre del año, pero cuando seleccionamos a las jugadoras internacionales, la sobrerrepresentación es mucho más marcada en el primer trimestre del año. Quizás con estos resultados, puede quedar un poco más claro que la selección por parte de los entrenadores, cuerpo técnico, de cara a alcanzar el alto rendimiento se hace con jugadoras nacidas en los primeros meses del año, pero si analizamos los datos antropométricos y condicionales queda reflejado, como veremos más adelante que al final del proceso evolutivo no se presentan diferencias en estas variables.

En relación a la lateralidad, la lateralidad de jugadoras de waterpolo no se encuentra efecto de la edad relativa ni en diestras ni en zurdas participantes en campeonatos del mundo (Barrenetxea-Garcia et al., 2018). En el balonmano alemán masculino (Schorer et al., 2009), se encuentra una sobrerrepresentación significativa de jugadores en el lateral izquierdo y derecho, así como en el extremo derecha. En este estudio se ha encontrado significación en las jugadoras diestras y no en las zurdas, una posible explicación puede ser que la población zurda presenta porcentajes bajos respecto a la diestra, y si a eso se añade que son jugadoras de balonmano, es posible no pasen por el mismo proceso de selección que las diestras, pues no suele haber competencia por ser seleccionadas.

Con respecto al puesto específico, se ha encontrado una sobrerrepresentación significativa de pivotes nacidas en el primer trimestre del año y una sobrerrepresentación de porteras nacidas en el primer semestre del año. En deporte femenino, se encuentran estudios en el fútbol español (Sedano et al., 2015) afirma que los entrenadores tienden a seleccionar a las defensas y porteras relativamente de más edad debido a su maduración física, estos resultados, a pesar de ser diferente deporte, pueden ser acordes a los obtenidos en nuestro estudio. En competiciones internacionales de balonmano masculinas de categorías inferiores, organizadas en categorías de dos años, se ha encontrado en los jugadores de segundo año, una sobrerrepresentación de jugadores de los dos primeros trimestres del año en todos los puestos específicos (Karcher et al., 2014).

En el presente estudio, no se han encontrado diferencias en variables antropométricas en relación al trimestre de nacimiento de las jugadoras de balonmano, de igual modo que tampoco las han encontrado en jugadoras de balonmano de 13 años de media al comparar entre semestres del año, ni en pliegues, ni circunferencias, ni longitudes y tampoco en variables básicas, como peso y altura (CamachoCardenosa et al., 2018). Por el contrario, en el género masculino si se encuentran diferencias. En jugadores de futbol ingleses entre 9 y 18 años se encuentran diferencias tanto en peso como en estatura, siendo 


\section{Cualidades físicas, técnicas y madurativas en balonmano femenino español}

mayores los valores en el primer trimestre que en el último del año (Lovell et al., 2015). En jugadores aficionados y de élite junior en Japón (Katsumata et al., 2018) también encuentran que los jugadores de primer trimestre del año los deportistas presentan mayores valores de altura y peso. En la élite masculina en un equipo de baloncesto del País Vasco muestran una edad cronológica mayor en los jugadores de la élite que en los aficionados, y mantienen mejores resultados en estatura y peso, igual que en los estudios anteriores (Torres-Unda et al., 2013). Estos resultados indican nuevamente que la competitividad en el deporte, mayor en categoría masculina que en la femenina, es un factor importante a la hora de explicar la presencia del efecto de la edad relativa.

Cuando se aborda la relación entre la condición física y el efecto de la edad relativa, encontramos en SJ un claro efecto de la edad relativa inverso basado en valores más elevados en el último trimestre del año, mientras que en CMJ no se aprecia efecto de la edad relativa (si bien se aprecia cierta tendencia a encontrar valora mayores en el segundo semestre, si bien no es una tendencia significativa) ni en la dinamometría máxima. En el presente estudio, los resultados de CMJ son similares a los obtenidos en jugadores masculinos de fútbol en Alemania entre 16 y 21 años de edad (Skorski, Faude, Hammes y Meyer, 2016), y de igual manera, similares a los encontrados en jugadores de fútbol masculinos de Bizkaia (España) de 10 años de edad, o en jugadores de tenis masculinos en Alemania (Ulbricht, Fernandez-Fernandez, Mendez-Villanueva, \& Ferrauti, 2015). Por el contrario, Muller, Muller, Hildebrandt, Kornexl, y Raschner (2015), encuentran en esquiadoras alpinas de 10 a 13 años los mayores valores en primer y cuarto trimestre del año. Pero en categorías inferiores del balonmano femenino se vuelven a observar valores similares y no significativo en el salto (Camacho-Cardenosa et al., 2018). A pesar de no existir muchos estudios que utilicen el SJ, los resultados, inicialmente difíciles de entender podrían explicarse, entendiendo que, aun sin significación estadística, las jugadoras del último trimestre del año son de menor altura y las que llegan a la élite son capaces de compensar su menor estatura con una mejor capacidad de salto.

Comparar aspectos técnico-tácticos específicos del balonmano, y concretamente velocidades de lanzamiento en diferentes situaciones de juego se torna especialmente difícil, aunque los resultados obtenidos no avalan una diferencia entre trimestres del año. Para la mejor interpretación de los resultados, hay que destacar que en el primer trimestre el $56 \%$ de las jugadoras son pivotes y porteras, ambos son puestos donde la variable velocidad de lanzamiento no es tan determinante, pues las pivotes realizan lanzamientos desde 6 metros o menos, donde los goles son más por la habilidad de lanzamiento que por la velocidad del mismo. $\mathrm{Y}$ en el caso de las porteras, no realizan la acción de lanzamiento habitualmente y por lo tanto se han eliminado del análisis. Mientras que el segundo trimestre se encuentra el mayor porcentaje de jugadoras de primera línea (centrales y laterales), donde realizan lanzamientos desde 9 metros, por lo que la velocidad de lanzamiento es muy importante.

\section{CONCLUSIONES}

Se ha encontrado un efecto de la edad relativa en jugadoras de balonmano españolas, mucho más marcado y soportado por las jugadoras que han participado en selecciones nacionales de distintas categorías de edad.

Cuando se valora la lateralidad encontramos un claro efecto de la edad relativa sólo en jugadoras diestras. Y por puestos específicos únicamente en pivotes y porteras.

No se ha encontrado efecto de la edad relativa en variables antropométricas, si bien si se ha encontrado en una variable condicional como es el SJ.

Finalmente, en variables técnico tácticas y en el resto de variables condicionales se han encontrado algunas diferencias que no pueden ser directamente achacables a la existencia de un efecto de la edad relativa.

\section{APLICACIONES PRÁCTICAS}

Existen varias estrategias para intentar paliar el efecto de la edad relativa, en el balonmano, y en general en los deportes de equipo. La primera, que los entrenadores, encargados de la detección y el desarrollo del talento deportivo estén al tanto y consideren el efecto de la edad relativa y que reaccionen para evitar dicho efecto en los procesos de selección, como por ejemplo en las competiciones internacionales de categorías inferiores (Bjorndal et al., 2018). La segunda, remarca la necesidad de un 


\section{Ferragut et al.}

cambio en las categorías de edad en competiciones internacionales que permita que los jugadores mayores puedan cambiar de categoría todos los años en lugar de cada ciclo de dos años. Si los formadores aplicasen este tipo de estrategias, el efecto de la edad relativa podría paliarse en gran medida y crear mayor igualdad de oportunidades entre los deportistas con independencia de su fecha de nacimiento.

\section{REFERENCIAS}

1. Abbott, A., \& Collins, D. (2004). Eliminating the dichotomy between theory and practice in talent identification and development: considering the role of psychology. Journal of Sports Sciences, 22(5), 395408. https://doi.org/10.1080/02640410410001675324

2. Albuquerque, M. R. (2013). Relative age effect in Olympic Judo athletes: A weight category analysis. $\begin{array}{llll}\text { Science \& } & \text { sports, } 28(3), & \text { e59. }\end{array}$ https://doi.org/10.1016/j.scispo.2012.09.004

3. Albuquerque, M. R., Lage, G. M., da Costa, V. T., Ferreira, R. M., Penna, E. M., Couto de Albuquerque Moraes, L. C. \& Malloy-Diniz, L. F. (2012). Relative age effect in olympic taekowndo athletes. Perceptual and Motor Skills, 114(2), 461-468. https://doi.org/ 10.2466/05.25.pms.114.2.461-468

4. Arrieta, H., Torres-Unda, J., Gil, M. \& Irazusta, J. (2016). Relative age effect and performance in the U16, U18 and U20 European Basketball Championships. Journal of Sports Sciences, 34(16), 1530-1534. https://doi.org/10.1080/02640414.2015.1122204

5. Ato, M., Lopez, J. J. \& Benavente, A. (2013). A classification system for research designs in psychology. Anales De Psicologia, 29(3), 1038-1059. https://doi.org/10.6018/analesps.29.3.178511

6. Barrenetxea-Garcia, J., Torres-Unda, J., Esain, I. \& Gil, S. M. (2018). Relative age effect and left-handedness in world class water polo male and female players. Laterality, $1-15$. https://doi.org/10.1080/1357650x.2018.1482906

7. Beals, T. C., Furtado, O. \& Fontana, F. (2012). The Relative Age Effect and Academic Timing in College Baseball. Research Quarterly for Exercise and Sport, 83, A32-A32.

8. Bjorndal, C. T., Luteberget, L. S., Till, K. \& Holm, S. (2018). The relative age effect in selection to international team matches in Norwegian handball. Plos One, 13(12). https://doi.org/10.1371/journal.pone.0209288

9. Bosco, C., Luhtanen, P. \& Komi, P. V. (1983). A simple method for measurement of mechanical power in jumping. European Journal of Applied Physiology and Occupational Physiology, 50(2), 273-282. https://doi.org/10.1007/bf00422166
10. Bošnjak, S. (2001). The declaration of Helsinki: the cornerstone of research ethics. Archive of Oncology, 9(3), 179-184.

11. Brazo-Sayavera, J., Martinez-Valencia, M. A., Muller, L., Andronikos, G. \& Martindale, R. J. J. (2017). Identifying talented track and field athletes: The impact of relative age effect on selection to the Spanish National Athletics Federation training camps. Journal of Sports Sciences, 35(22), 2172-2178. https://doi.org/10.1080/02640414.2016.1260151

12. Brustio, P. R., Lupo, C., Ungureanu, A. N., Frati, R., Rainoldi, A. \& Boccia, G. (2018). The relative age effect is larger in Italian soccer top-level youth categories and smaller in Serie A. Plos One, 13(4). https://doi.org/10.1371/journal.pone.0196253

13. Camacho-Cardeñosa, A., Camacho-Cardenosa, M., Gonzalez-Custodio, A., Martinez-Guardado, I., Timon, R., Olcina, G. \& Brazo-Sayavera, J. (2018). Anthropometric and Physical Performance of Youth Handball Players: The Role of the Relative Age. Sports, 6(2). https://doi.org/10.3390/sports6020047

14. Carling, C., le Gall, F., Reilly, T. \& Williams, A. M. (2009). Do anthropometric and fitness characteristics vary according to birth date distribution in elite youth academy soccer players? Scandinavian Journal of Medicine \& Science in Sports, 19(1), 3-9. https://doi.org/10.1111/j.1600-0838.2008.00867.x

15. Carter, J. \& Heath, B. (1990). Somatotyping: development and applications. New York: Cambridge University Press.

16. Cervera-Raga, V., Jimenez-Saiz, S. \& Lorenzo-Calvo, A. (2013). The relative effect of age and gender in secondary schoool fitness testing. Revista De Psicologia Del Deporte, 22(2), 447-452.

17. Cohen, J. (1988). Statistical power analysis for the behavioral sciences (L. E. Associates Ed. 2nd Edition ed.). Hillsdale, N.J.: L. Erlbaum Associates.

18. de Dios Roman-Seco, J. (2015). historical update on handball game evolution throughout XXth Century. $E$ Balonmano: Revista de Ciencias del Deporte, 11(1), 3-38.

19. Delorme, N., Boiché, J. \& Raspaud, M. (2010). Relative age effect in female sport: a diachronic examination of soccer players. Scandinavian Journal of Medicine \& Science in Sports, 20(3), 509-515. https://doi.org/10.1111/j.1600-0838.2009.00979.x

20. Delorme, N. \& Champely, S. (2015). Relative Age Effect and chi-squared statistics. International Review for the Sociology of Sport, 50(6), 740-746. https://doi.org/10.1177/1012690213493104

21. Doblhammer, G. \& Vaupel, J. W. (2001). Lifespan depends on month of birth. Proceedings of the National Academy of Sciences of the United States of America, 98(5), 2934-2939. https://doi.org/10.1073/pnas.041431898 


\section{Cualidades físicas, técnicas y madurativas en balonmano femenino español}

22. Doyle, J. R. \& Bottomley, P. A. (2018). Relative age effect in elite soccer: More early-born players, but no better valued, and no paragon clubs or countries. Plos One,

13(2). https://doi.org/10.1371/journal.pone.0192209

23. Edgar, S. \& O'Donoghue, P. (2005). Season of birth distribution of elite tennis players. Journal of Sports Sciences, 23(10), 1013-1020. https://doi.org/10.1080/02640410400021468

24. Garson, G. D. (2010). Statnotes: Topics in multivariate analysis. Multiple regression: Residul analysis. Retrieved from https://faculty.chass.ncsu.edu/garson/PA765/statnote.h $\underline{\mathrm{tm}}$

25. Gutierrez-Diaz-del-Campo, D., Pastor-Vicedo, J. C., Gonzalez-Villora, S. \& Contreras-Jordan, O. R. (2010). The relative age effect in youth soccer players from Spain. Journal of Sports Science and Medicine, 9(2), 190-198. https://doi.org/10.3390/sports5010005

26. Gómez-López, M., Angosto-Sánchez, S., RuízSánchez, V. \& Pérez-Turpín, J. A. (2017). Relative age effect in handball players of Spain. Journal of Physical Education and Sport, 17(2), 705-711. https://doi.org/10.7752/jpes.2017.02106

27. Hancock, D. J., Young, B. W. \& Ste-Marie, D. M. (2011). Effects of a rule change that eliminates bodychecking on the relative age effect in Ontario minor ice hockey. Journal of Sports Sciences, 29(13), 1399-1406. https://doi.org/10.1080/02640414.2011.593040

28. Hirose, N. (2009). Relationships among birth-month distribution, skeletal age and anthropometric characteristics in adolescent elite soccer players. Journal of Sports Sciences, 27(11), 1159-1166. https://doi.org/10.1080/02640410903225145

29. Jimenez, I. P. \& Pain, M. T. G. (2008). Relative age effect in Spanish association football: Its extent and implications for wasted potential. Journal of Sports Sciences, 26(10), 995-1003. https://doi.org/10.1080/02640410801910285

30. Karcher, C., Ahmaidi, S. \& Buchheit, M. (2014). Effecto of birth date on playing time during international handball competitions with respect to playing positions. Kinesiology, 46(1), 23-32.

31. Katsumata, Y., Omuro, K., Mitsukawa, N. \& Nakata, H. (2018). Characteristics of Relative Age Effects and Anthropometric Data in Japanese Recreational and Elite Male Junior Baseball Players. Sports medicine open, 4(1), 52-52. https://doi.org/10.1186/s40798-0180165-9

32. Korgaokar, A., Caputo, J. L., Farley, R. S. \& Fuller, D. K. (2014). Relative Age Effect Among Elite Youth Male Soccer Players Across the United States. Medicine and Science in Sports and Exercise, 46(5), 956-956.

https://doi.org/10.1249/01.mss.0000496372.63459.d7
33. Lee, R. C., Wang, Z. M., Heo, M. S., Ross, R., Janssen, I. \& Heymsfield, S. B. (2000). Total-body skeletal muscle mass: development and cross-validation of anthropometric prediction models. American Journal of Clinical Nutrition, 72(3), 796-803. https://doi.org/10.1093/ajcn/72.3.796

34. Leite, N., Borges, J., Santos, S. \& Sampaio, J. (2013). The relative age effect in school and federative sport in basketball. Revista De Psicologia Del Deporte, 22(1), 219-222.

35. Lidor, R., Arnon, M., Maayan, Z., Gershon, T. \& Côté, J. (2014). Relative age effect and birthplace effect in Division 1 female ballgame players - the relevance of sport-specific factors. International Journal of Sport and Exercise Psychology, 12(1), 19-33. https://doi.org/10.1080/1612197X.2012.756232

36. Loffing, F., Schorer, J. \& Cobley, S. P. (2010). Relative Age Effects are a developmental problem in tennis: but not necessarily when you're left-handed! High Ability Studies, 21(1), 19-25. https://doi.org/10.1080/13598139.2010.488084

37. Lovell, R., Towlson, C., Parkin, G., Portas, M., Vaeyens, R. \& Cobley, S. (2015). Soccer Player Characteristics in English Lower-League Development Programmes: The Relationships between Relative Age, Maturation, Anthropometry and Physical Fitness. Plos One, 10(9). https://doi.org/10.1371/journal.pone.0137238

38. Lupo, C., Boccia, G., Ungureanu, A. N., Frati, R., Marocco, R. \& Brustio, P. R. (2019). The Beginning of Senior Career in Team Sport Is Affected by Relative Age Effect. Frontiers in Psychology, 10. https://doi.org/10.3389/fpsyg.2019.01465

39. Melchiorri, G., Viero, V., Triossi, T., Annino, G., Padua, E. \& Tancredi, V. (2017). Anthropometric and performance measures $\mathrm{t}$ o study talent detection in youth volleyball. Journal of Sports Medicine and Physical Fitness, 57(12), 1623-1632. https://doi.org/10.23736/s0022-4707.17.07068-2

40. Mujika, I., Vaeyens, R., Matthys, S. P. J., Santisteban, J., Goiriena, J. \& Philippaerts, R. (2009). The relative age effect in a professional football club setting. Journal of Sports Sciences, 27(11), 1153-1158. https://doi.org/10.1080/02640410903220328

41. Muller, L., Muller, E., Hildebrandt, C., Kornexl, E. \& Raschner, C. (2015). Influential Factors on the Relative Age Effect in Alpine Ski Racing. Plos One, 10(8). https://doi.org/10.1371/journal.pone.0134744

42. Nakata, H. \& Sakamoto, K. (2011). Relative age effect in Japanese male athletes. Percept Mot Skills, 113(2), 570-574. https://doi.org/10.2466/05.10.11.PMS.113.5.570-574

43. Ortigosa-Marquez, J. M., Reigal, R. E., Serpa, S. \& Hernandez-Mendo, A. (2018). Relative age effect on national selection proceess in triathlon. Revista 


\section{Ferragut et al.}

Internacional de Medicina y Ciencias de la Actividad Fisica y del Deporte, 18(70), 199-211. https://doi.org/10.15366/rimcafd2018.70.001

44. Palao, J. M., Manzanares, P. \& Valades, D. (2014). Anthropometric, Physical, and Age Differences by the Player Position and the Performance Level in Volleyball. Journal of Human Kinetics, 44(1), 223236. https://doi.org/10.2478/hukin-2014-0128

45. Papadopoulou, S. D., Papadopoulou, S. K., Rosemann, T., Knechtle, B. \& Nikolaidis, P. T. (2019). Relative Age Effect on Youth Female Volleyball Players: A Pilot Study on Its Prevalence and Relationship With Anthropometric and Physiological Characteristics. Frontiers in psychology, 10, 2737-2737. https://doi.org/10.3389/fpsyg.2019.02737

46. Penna, E. M., Campos, B. T., Gonçalves, G. G. P., Godinho, G. H. P., Lima, C. O. V. \& Prado, L. S. (2018). Relative age effect and dropout causes in a multisport club setting. Is there a special reason to give up? Motriz: Revista de Educação Física, 24(4), e101801-e101801. https://doi.org/10.1590/s19806574201800040011

47. Ross, W. D. \& Marfell-Jones, R. J. (1995). Cineantropometría. In J. Duncan, H. MacDougall, A. Wenger, \& H. J. Green (Eds.), Evaluación fisiológica del deportista. Barcelona: Paidotribo.

48. Saavedra-García, M.A., Gutiérrez-Aguilar, O., Galatti, L. y Fernández-Romero, J. J. (2015). Efecto de la edad relativa en los mundiales de baloncesto FIBA en cateogrías inferiores (1979-2011). Cuadernos de Psicologia del Deporte, 15(3). https://doi.org/10.4321/s1578-84232015000300028

49. Saavedra-Garcia, M., Matabuena, M., MonteroSeoane, A. \& Fernandez-Romero, J. J. (2019). A new approach to study the relative age effect with the use of additive logistic regression models: A case of study of FIFA football tournaments (1908-2012). PloS one, 14(7), e0219757-e0219757. https://doi.org/10.1371/journal.pone.0219757

50. Saavedra-Garcia, M. A., Gutiérrez-Aguilar, O., Fernández-Romero, J. J., Fernández-Lastra, D. \& Eiras-Oliveira, G. (2014). Relative age effect in lower categories of international basketball. International Review for the Sociology of Sport, 49(5), 526-535. https://doi.org/10.1177/1012690212462832

51. Saavedra-García, M., Gutiérrez-Aguilar, O., SaMarques, P. y Fernández-Romero, J. J. (2016). Efecto de la edad relativa en el atletismo español. Cuadernos de Psicología del Deporte, 16(1), 275-286. https://doi.org/10.4321/s1578-84232015000300028

52. Schorer, J., Cobley, S., Buesch, D., Braeutigam, H., Baker, J., Büsch, D. \& Bräutigam, H. (2009). Influences of competition level, gender, player nationality, career stage and playing position on relative age effects. Scandinavian journal of medicine \& science in sports, 19(5), 720-730. https://doi.org/10.1111/j.1600-0838.2008.00838.x

53. Schorer, J., Wattie, N. \& Baker, J. R. (2013). A New Dimension to Relative Age Effects: Constant Year Effects in German Youth Handball. Plos One, 8(4). https://doi.org/10.1371/journal.pone.0060336

54. Sedano, S., Vaeyens, R. \& Redondo, J. C. (2015). The Relative Age Effect in Spanish Female Soccer Players. Influence of the Competitive Level and a Playing Position. Journal of Human Kinetics, 46(1), 129-137. https://doi.org/10.1515/hukin-2015-0041

55. Skorski, S., Faude, O., Hammes, D. \& Meyer, T. (2016). The Relative Age Effect in Elite German Youth Soccer: Implications for a Successful Career. International Journal of Sports Physiology and Performance, 11(3), 370-376. https://doi.org/10.1123/ijspp.2015-0071

56. Torres-Unda, J., Zarrazquin, I., Gil, J., Ruiz, F., Irazusta, A., Kortajarena, M., Seco, J. \& Irazusta, J. (2013). Anthropometric, physiological and maturational characteristics in selected elite and nonelite male adolescent basketball players. Journal of Sports Sciences, 31(2), 196-203. https://doi.org/10.1080/02640414.2012.725133

57. Tyebkhan, G. (2003). Declaration of Helsinki: the ethical cornerstone of human clinical research. Indian Journal of Dermatology, Venereology and Leprology, 69(3), 245-247.

58. Ulbricht, A., Fernandez-Fernandez, J., MendezVillanueva, A. \& Ferrauti, A. (2015). The Relative Age Effect and Physical Fitness Characteristics in German Male Tennis Players. Journal of Sports Science and Medicine, 14(3), 634-642.

59. WMA. (2009). Declaration of Helsinki. Ethical principles for medical research involving human subjects. Journal of the Indian Medical Association, 107(6), 403-405. 